\title{
Hamiltonian triangular refinements and space-filling curves
}

\author{
Alberto Márquez a , Ángel Plaza ${ }^{\mathrm{b}, \mathrm{c}}$, José P. Suárez ${ }^{\mathrm{c}, *}$ \\ a Department of Applied Mathematics I, University of Seville, Spain \\ ${ }^{\mathrm{b}}$ Department of Mathematics, University of Las Palmas de Gran Canaria, Spain \\ c Division of Mathematics, Graphics and Computation, IUMA, Information and Communication Systems, University of Las Palmas de Gran Canaria, Spain
}

Keywords:

Hamiltonian triangulations Space-filling curve Mesh refinement Longest edge

\begin{abstract}
We have introduced here the concept of Hamiltonian triangular refinement. For any Hamiltonian triangulation it is shown that there is a refinement which is also a Hamiltonian triangulation and the corresponding Hamiltonian path preserves the nesting condition of the corresponding space-filling curve. We have proved that the number of such Hamiltonian triangular refinements is bounded from below and from above. The relation between Hamiltonian triangular refinements and space-filling curves is also explored and explained.
\end{abstract}

\section{Introduction}

Numerical mesh generation and refinement of a given mesh are the main steps in many areas such as Computational Geometry, Computer Graphics, Geometric Modeling or the Finite Element Method (FEM). On the other hand, there is a significant interest for using space-filling curves (SFC) in scientific and engineering applications. A space-filling curve is a continuous mapping from the one-dimensional space onto the multiple-dimensional space.

Hilbert recognized a general geometrical generating procedure that allowed the construction of an entire class of spacefilling curves [1]. Hilbert promulgated the following heuristic principle: If the interval $\mathcal{I}=[0,1]$ can be mapped continuously onto the square $\mathcal{Q}=[0,1]^{2}$, then after partitioning $\mathcal{I}$ into four congruent subintervals and $\mathcal{Q}$ into four congruent sub-squares, each subinterval can be mapped continuously onto one of the sub-squares. Next, each subinterval is, in turn, partitioned into four congruent subintervals and each sub-square into congruent sub-squares, and the argument is repeated. If this is carried on ad infinitum, $\mathcal{I}$ and $\mathcal{Q}$ are partitioned into $2^{2 n}$ congruent replicas for $n=1,2,3 \ldots$

Hilbert demonstrated that the sub-squares can be arranged so that adjacent subintervals correspond to adjacent subsquares with an edge in common, and so that the inclusion relationships are preserved, i.e., if a square corresponds to an interval, then its sub-squares correspond to the subintervals of that interval (nesting condition) [2]. See Fig. 1. Both the Hilbert curve and its discrete approximations are useful because they give a mapping between $1 D$ and $2 D$ space that fairly well preserves locality [3]. If $(x, y)$ are the coordinates of a point within the unit square, and $\mathrm{d}$ is the distance along the curve when it reaches that point, then points that have nearby $d$ values will also have nearby $(x, y)$ values. The converse cannot always be true. There will sometimes be points where the $(x, y)$ coordinates are close but their $d$ values are far apart.

\footnotetext{
* Correspondence to: University of Las Palmas de Gran Canaria, 35017 Las Palmas de Gran Canaria, Spain.

E-mail address: josepablo.suarez@ulpgc.es (J.P. Suárez).
} 


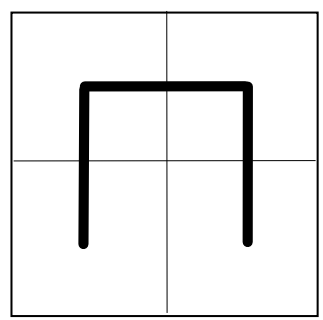

Resolution 1

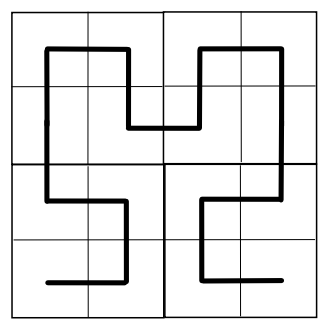

Resolution 2

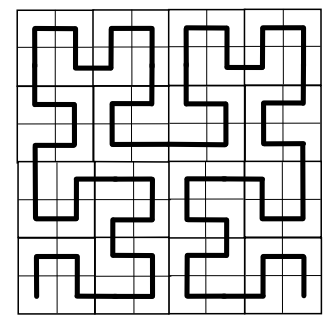

Resolution 3

Fig. 1. First steps in the generation of Hilbert's Curve.
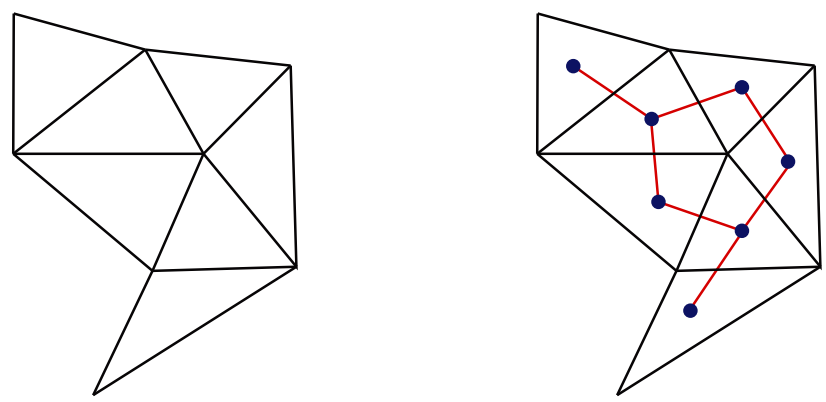

Fig. 2. (Left) Non-Hamiltonian triangulation and (right) its dual graph.

Of special interest is the connection of SFC's to mesh generation. Obviously, if a mesh can be described by a path traversing all the triangles (or quadrilaterals in the case of quadrangulations) only once, then that given mesh can be stored and processed in a very efficient way. This is the idea behind Hamiltonian triangulations.

A Hamiltonian path is a path in an undirected or directed graph that visits each vertex exactly once. A triangulation is called a Hamiltonian triangulation if its dual graph deleting the vertex corresponding to the outer face (the inner dual) admits a Hamiltonian path. In other words, there is a path that visits each vertex exactly once in the dual graph of the triangulation, that is the path visits each triangle exactly once. In other case, a triangulation is called non-Hamiltonian. A simple example of non-Hamiltonian triangulation is given in Fig. 2.

Hamiltonian properties of triangulations have been studied extensively, see for example [4-6]. Space-filling curves are in the background of some of the latest proposed data structures [7] in the area of digital terrain representations [8,9] and image compression [10].

Even in the field of mesh refinement and associated data structures SFC's have shown their applicability [11,12].

We introduce here the concept of Hamiltonian triangular refinement. For any Hamiltonian triangulation it is shown that there is a refinement which results in a Hamiltonian triangulation and also preserves the nesting condition of the corresponding Hamiltonian path. The number of such Hamiltonian triangular refinements is bounded from below and from above. We prove the number of Hamiltonian refinements (that depends on the number of triangles and on Fibonacci numbers) yielding Hamiltonian triangulation.

It should be noted that our scenario does not uniquely apply to initial triangulations containing a unique Hamiltonian path. We may consider an initial triangulation where more than one Hamiltonian path are included. In this case, the Hamiltonian refinement when applied to such triangulation preserves the same number of Hamiltonian paths as in the initial triangulation. If the existence of a single Hamiltonian path is relevant to the problem of interest, for example in geometric compression and transmission, then there is a solution proposed in [4] where a given triangulated model can be represented as a single triangle strip (Hamiltonian path).

Fig. 3(a) shows a simple Hamiltonian triangulation and its refinement which is also Hamiltonian, Fig. 3(b).

\section{Refinement of a triangulation and triangle partition}

Mesh refinement is often divided into two types: uniform and local. Uniform implies the refinement of all triangles in a mesh, usually following a specified local subdivision pattern. If the refinement is made locally for only a single triangle or a sub-group of triangles, then the refinement is local. Local refinement of triangular meshes involves two main tasks. The first is the local partition of the target triangles and the second is the propagation to successive neighbor triangles to preserve mesh conformity. Several approaches for partitioning triangles have been studied. 


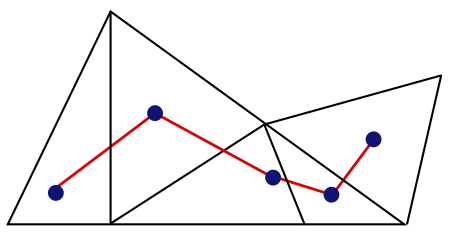

(a)

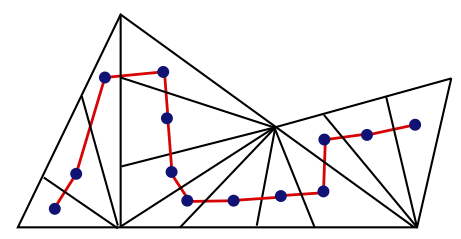

(b)

Fig. 3. (a) Hamiltonian triangulation and (b) Hamiltonian refinement.

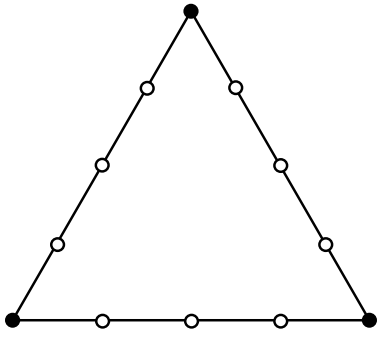

(a)

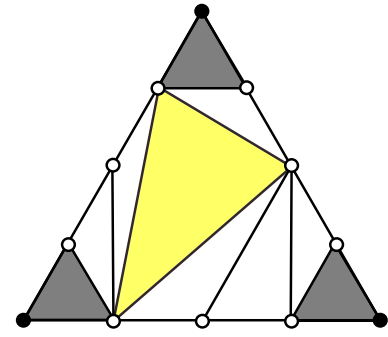

(b)

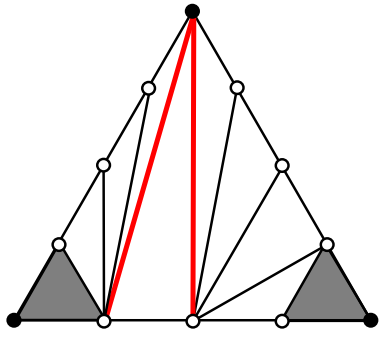

(c)

Fig. 4. $k$-partition of a triangle, interior triangle (in yellow) and ears (in gray). Two corner-edge diagonals are colored in red in (c). (For interpretation of the references to color in this figure legend, the reader is referred to the web version of this article.)

A triangulation of a finite planar point set $S$ is a dissection of its convex hull by non-crossing diagonals into triangles. In the $2 \mathrm{D}$ plane triangulations are made up of triangles, together with their edges and vertices. A triangulation is conforming if any adjacent triangle share an entire edge or a common vertex, i.e. there are no hanging nodes. Here we will consider only conforming triangulations where the vertices of all the triangles are non collinear points. Conforming triangulations are usually employed in the Finite Element context $[13,14]$.

Definition 1 ( $k$-Partition of a Triangle). For a triangle $t$ with vertices $A B C$ let $S_{k}$ be the set of points $A, B, C$ and $k$ equality spaced points in each edge of triangle $t$. That is, $S_{k}=\left\{A, B, C, A_{1}, \ldots, A_{k}, B_{1}, \ldots, B_{k}, C_{1}, \ldots, C_{k}\right\}$, where $A_{i}, B_{i}, C_{i}$ are $k$ equally spaced points respectively in edges $a, b$ and $c$. A $k$-partition of $t$ is any triangulation of $S_{k}$.

Notice that in order to avoid trivial situations, we will consider $k \geq 1$, since for $k=0$ it is obtained the trivial triangulation consisting only in the initial triangle. Then in any $k$-partition of a triangle with vertices $A B C$ each edge of the triangle is subdivided into the same number of parts $k+1$, see Fig. 4(a). Let $T$ be a $k$-partition of triangle $A B C$. A corner-edge diagonal is a diagonal of $T$ one of whose endpoints is a corner of $A B C$ and the other is an interior point of the opposite edge [15]. Two corner-edge diagonals are in the triangulation in Fig. 4(c), colored in red. On the other hand, the triangulation in Fig. 4(b) does not contain any corner-edge diagonal. Let us consider a vertex of the triangle, say $A$. Then either there is at least an interior corner-edge diagonal at $A$ or there is not such interior diagonal. Notice that, according to the definition, there might be multiple corner-edge diagonals. Since we consider only conforming triangulation, if there are more than one corner-edge diagonal, all of them share a corner of the initial triangle with vertices $A B C$.

If in vertex $A$ there is not an interior diagonal, then there is a triangle in the $k$-partition of triangle $A B C$ with vertex $A$, two edges in edges $b, c$ of triangle $A B C$ sharing $A$ and the third edge interior to triangle $A B C$. This triangle of the $k$-partition is called an ear. For example, the triangulation in Fig. 4(b) has ears in all three vertices, while the triangulation in Fig. 4(c) has two ears. All the ears are marked in gray color.

An interior triangle is a triangle of $T$ whose vertices are interior points of different edges of $A B C$. Notice that if there is an interior triangle in a triangulation, by removing that triangle, there will appear three non-connected subtriangulations. In other words, an interior triangle has valence 3 in the dual graph. Therefore, if a triangulation of a single triangle $A B C$ has an interior triangle, the triangulation is not Hamiltonian. For example, the triangulation in Fig. 4(b) contains a central triangle (in yellow in the figure), while the triangulation in Fig. 4(c) has not a central triangle. A regular triangle is a triangle which is neither an ear nor an interior triangle. Notice that a regular triangle has valence 2 in the dual graph.

More precisely let $T$ be a $k$-triangulation of $A B C$. Then either $T$ has one central triangle, three ears, and no corneredge diagonal, or $T$ has no central triangle, two ears, and at least one corner-edge diagonal emanating from the remaining corner. Triangulations of the former kind will be called non-Hamiltonian or $n H_{k}$-triangulations (see Fig. 5(a) for an example), and triangulations of the latter kind will be called Hamiltonian or $H_{k}$-triangulations (see Fig. 5(b) for an example). We are interested here on the number of $H_{k}$-triangulations. Obviously, since the partition of the triangle has to be a Hamiltonian 


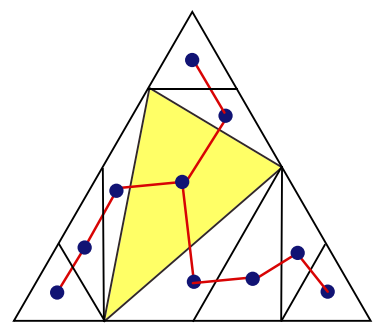

(a)

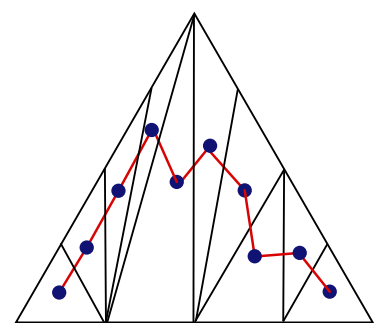

(b)

Fig. 5. Dual graphs for triangulations in Fig. 4.
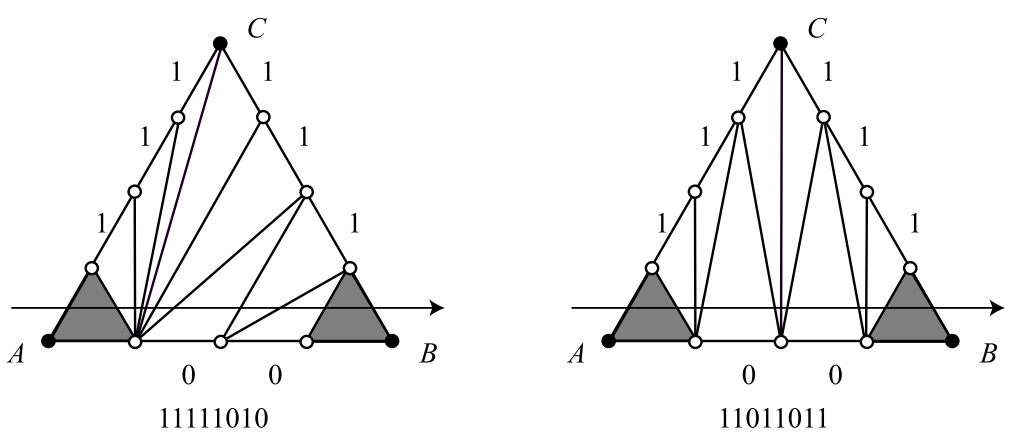

Fig. 6. Two $H_{k}$-triangulations and the associated 01 codes.

triangulation, then its dual graph is a path and it will present exactly two ear triangles at one of the edges of the parent triangle.

Proposition 2. In a triangle, there are $\left(\begin{array}{c}3 k \\ k\end{array}\right) H_{k}$-triangulations.

Proof. This proposition is a particular case of [15, Theorem 8]. Here we use an encoding of triangulations by $\{0,1\}$-sequences similar to that from $[15,16]$.

The definition and construction algorithm of sequence $\{0,1\}$ follows: Let $T$ be an $H_{k}$-triangulation, of triangle with vertices $A B C$. As far as any $H_{k}$-triangulation, say $T$, has two ears, these two ears define an edge in the parent triangle. Let $C B$ be the edge in the parent triangle $t$ defined by the two ears as in Fig. 6. Each regular triangle of $T$ shares exactly one edge with one of the edges of $A B C$. We encode the regular triangles that share an edge with $C B$ by 0 , and those that share an edge with the other two edges, $A C$ or $A B$, by 1 . Consider the directed segment $C B$, and shift it slightly ("infinitesimally") into the interior of triangle $A B C$. The segment obtained in that way intersects all the triangles of $T$ and consequently induces a linear order on them. The $\{0,1\}$-sequence associated to triangulation $T$ is just the sequence of 0 's and 1's corresponding to the triangles as traversed by that shifted segment.

Using the linear order that was described above, we obtain a $\{0,1\}$-sequence of length $3 k-1$, in which 0 occurs $k-1$ times and 1 occurs $2 k$ times. See Fig. 6 for an illustration of two $H_{k}$-triangulations and their $\{0,1\}$-sequences. It is easy to see that this correspondence between $H_{k}$-triangulations of $S_{k}$ and $\{0,1\}$-sequences with $k-1$ occurrences of 0 and $2 k$ occurrences of 1 is bijective.

Since there are 3 possibilities for the vertex with no ear, the total number of $H_{k}$-triangulations of triangle $t$ is

$$
3\left(\begin{array}{c}
3 k-1 \\
k-1
\end{array}\right)=3 \cdot \frac{(3 k-1) !}{(k-1) !(2 k) !}=\frac{3 k(3 k-1) !}{k(k-1) !(2 k) !}=\left(\begin{array}{c}
3 k \\
k
\end{array}\right) \text {. }
$$

\section{Hamiltonian refinement of a Hamiltonian triangulation}

In this section we will focus on Hamiltonian triangulations, that is triangulations admitting at least one Hamiltonian path, and we will prove that any of them admits refinements that are also Hamiltonian.

Definition 3 (Nesting Condition). Let $\tau$ be a given triangulation with Hamiltonian path $H$. Let $\tau^{\prime}$ be a triangular refinement of $\tau$, so each triangle $t_{j} \in \tau$ is subdivided in $n_{j}$ subtriangles. A Hamiltonian path $H^{\prime}$ of $\tau^{\prime}$ holds de nesting condition with respect 


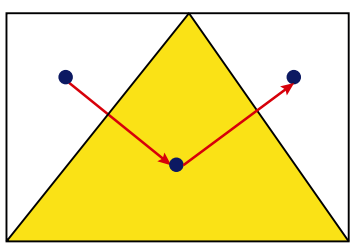

(a)

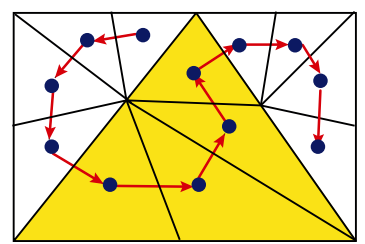

(b)

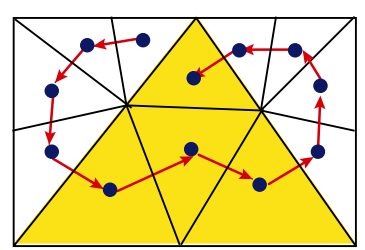

(c)

Fig. 7. (a) Hamiltonian triangulation with its Hamiltonian path, (b) Hamiltonian refinement of the original path, and (c) Hamiltonian path of a refinement without the nesting condition.

to $H$ if: If subinterval $I_{j}$ corresponds to subtriangle $t_{j} \in \tau$ by $H$, then there are $n_{j}$ subintervals of $I_{j}$ that correspond to the $n_{j}$ subtriangles of $t_{j}$ by $H^{\prime}$.

Definition 4 ( $k$-Mapping for a Given Hamiltonian Triangulation $\tau$ ). Let $k$ be a positive integer, and $\tau$ a given triangulation. Let $\mathcal{C}$ be the set of $H_{k}$-triangulations, a mapping $f: \tau \longrightarrow \mathcal{C}$ is called a $k$-mapping for $\tau$.

Notice that $f$ maps the set of triangles to the set of $H_{k}$-triangulations.

Definition 5 ( $f$-Refinement of a Triangulation $\tau)$. Let $\tau$ be a triangulation, and let $f$ be a $k$-mapping for $\tau$. $f(\tau)$ is a refinement of $\tau$ such that the refinement of each triangle $T \in \tau$ is the $H_{k}$-triangulation determined by $f$ and is called a $f$-refinement of $\tau$.

Remark 6. For any triangle $T \in \tau$ there are three $k$-partitions of $T$ determined by $f(T)$ since there are 3 edges where the ears can be put. Then, if $n$ is the number of triangles of $\tau$, there are $3^{n} f$-refinements of $\tau$.

Definition 7 (Hamiltonian $f$-Refinement of a Hamiltonian Path). Let $\tau$ be a Hamiltonian triangulation with Hamiltonian path $H_{\tau}$, and let $f$ be a $k$-mapping for $\tau$. A $f$-refinement $\tau^{\prime}$ of $\tau$ is called a Hamiltonian refinement of $H_{\tau}$ if it satisfies the following three conditions:

(1) The refinement of each triangle $T \in \tau$ is the $k$-partition determined by $f$.

(2) $\tau^{\prime}$ admits a Hamiltonian path $H_{\tau^{\prime}}$.

(3) $H_{\tau^{\prime}}$ holds the nesting condition with respect to $H_{\tau}$.

Fig. 7 shows a simple Hamiltonian triangulation and two refinements. The first one, see Fig. 7(b), is a Hamiltonian refinement while the second one, Fig. 7(c) is not because there is no a Hamiltonian path holding the nesting property with respect to the initial triangulation and Hamiltonian path in Fig. 7(a).

Theorem 8 (Number of Hamiltonian $f$-Refinements). Let $\tau$ be a Hamiltonian triangulation with $n \geq 3$ triangles and with Hamiltonian path $H_{\tau}$, let $f$ be a $k$-mapping for triangulation $\tau$. Then, $H_{\tau}$ always admits a Hamiltonian refinement. Even more, if $c_{\tau}$ represents the number of Hamiltonian $f$-refinements of $H_{\tau}$, then $2(3+n) \leq c_{\tau} \leq 4 F_{n+1}$, where $F_{n}$ represents the nth Fibonacci number. ${ }^{1}$

Proof. Observe that in any Hamiltonian refinement when the Hamiltonian path crosses through a triangle, there are three possible positions of the $k$-partition (the two ears of the $k$-partition define one of the edges, so we have one position for each edge). We can identify each valid partition in a triangle $A B C$ of $\tau$ by the two vertices where the ears are located.

Now, we are going to define a digraph $D_{\tau}$ associated to $H_{\tau}$ in such a way that each Hamiltonian refinement $R_{\tau}$ is going to be represented by a maximal directed path in that digraph.

\footnotetext{
1 The Fibonacci numbers are defined by $F_{0}=0, F_{1}=1$ and the recurrence relation $F_{n}=F_{n-1}+F_{n-2}$ for $n \geq 2$.
} 

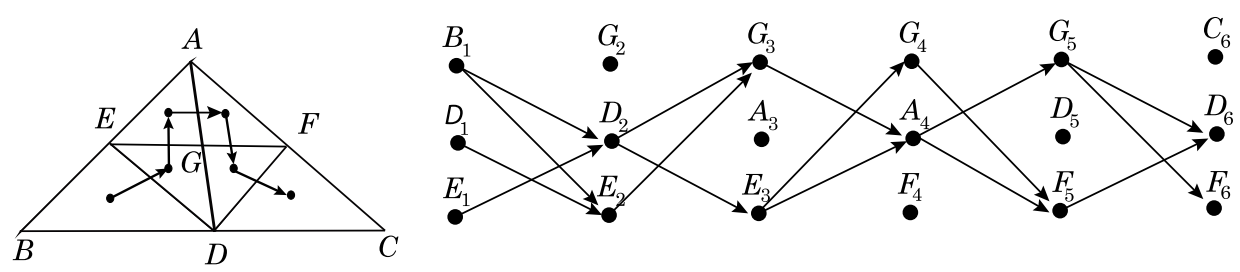

Fig. 8. (Left) A Hamiltonian triangulation and its Hamiltonian path. (Right) The digraph associated.
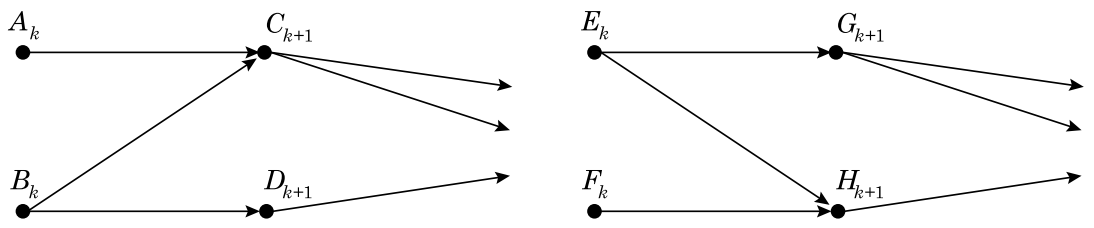

Fig. 9. (Left) Two arrows from level $k$ to level $k+1$ arrive to the vertex with outdegree 2. (Right) Two arrows from level $k$ to level $k+1$ arrive to the vertex with outdegree 1.
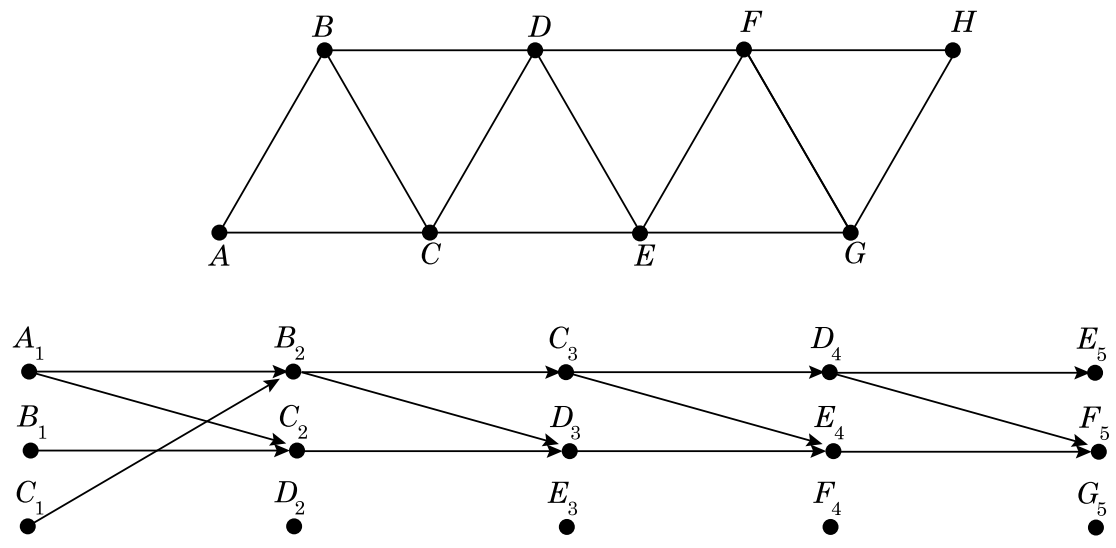

Fig. 10. The triangulation giving the lower bound of Theorem 8 and its associated digraph.

The number of vertices in $D_{\tau}$ is three times the number of triangles in $\tau$, so we can represent those vertices sorted in layers of three vertices (one for each triangle of the path $H_{\tau}$ ) each one corresponding to the three vertices of each triangle. For the sake of clarity, vertex $A$ in level $k$ will be represented by $A_{k}$ (and that means that $A$ is a vertex in the $k$ th triangle in the path $H_{\tau}$ ). The edges run from layer $k$ to layer $k+1$. We start representing the edges from level 1 to level 2 , we add an edge from a vertex $A_{1}$ to a vertex $B_{2}$ if $A \neq B$ and $B$ is a vertex in the first triangle and in the second triangle of $H_{\tau}$. Thus, we have four edges from level 1 to level 2 . In general, we add one edge from vertex $A_{k}$ in level $k$ to vertex $B_{k+1}$ in level $k+1$ if there exists an edge in the previous level finishing at $A_{k}$, and $B$ is a vertex shared by the $k$ th triangle and the $(k+1)$-st triangle other than $A$. Thus, in each layer one of the vertices has outdegree 1 , other one has outdegree 2 , and the last one is isolated. The number of Hamiltonian refinements of $\tau$ can be identified, in this way, with twice the number of directed paths from the first to the last level of $D_{\tau}$. Note that we must double the number of paths since from any of the two non-isolated vertices of the last level we can draw two outgoing edges. Fig. 8 shows a Hamiltonian triangulation, its Hamiltonian path and the digraph associated.

From layer $k$ to layer $k+1$ there are two possible schemes depicted in Fig. 9. If we denote by $x_{k}$ the number of paths arriving to the vertex with outdegree 1 in the level $k$ and by $y_{k}$ the number of paths arriving to the vertex with outdegree 2 , it is clear that the first scheme leads to: $x_{k+1}=y_{k}$ and $y_{k+1}=x_{k}+y_{k}$, and the second scheme leads to $x_{k+1}=x_{k}+y_{k}$ and $y_{k+1}=y_{k}$, and we can consider $x_{1}=2$ and $y_{1}=2$. So, we can determine the number of refinements via a simple matrix multiplication.

Finally, in the last triangle, we can consider two edges going out from each one of the two vertices with indegree greater than zero.

Then, the two extreme cases are that we have only the first scheme and we obtain the lower bound (see Fig. 10) that appears in the statement of the theorem, and if we have only the second scheme, we obtain the upper bound (see Fig. 11). 

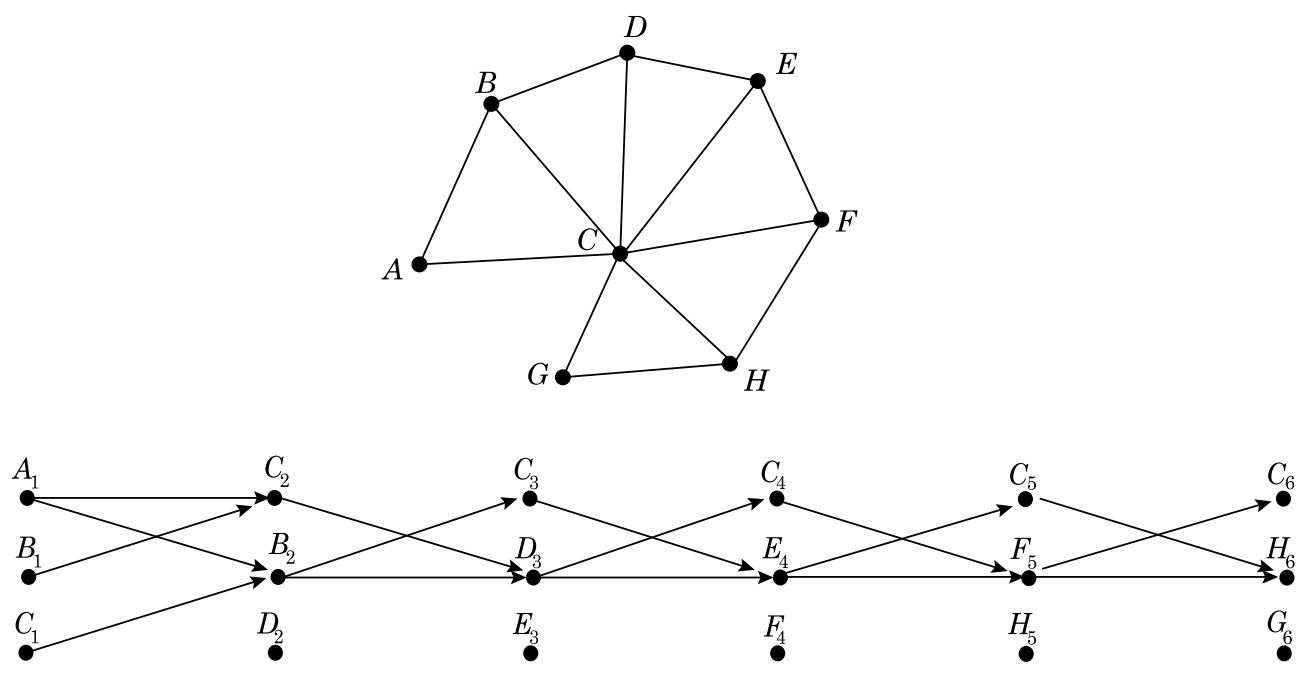

Fig. 11. The triangulation giving the upper bound of Theorem 8 and its associated digraph.
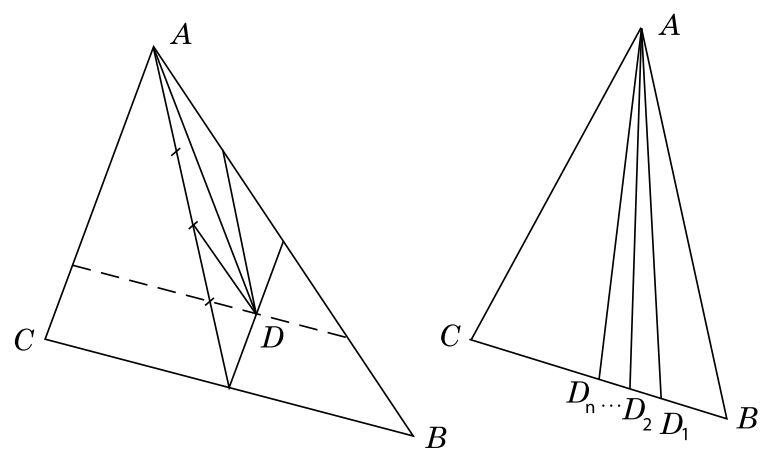

Fig. 12. (Left) The distance from $A$ to $D$ is smaller than three quarters of the distance from $A$ to $B$, (right) the distance from $A$ to successive points $D$ converges to the height of the triangle.

\section{Space-filling curves from Hamiltonian triangulations}

If we start in a Hamiltonian triangulation and we refine it an infinite number of times, it is not clear that we always obtain a SFC from the limit of the sequence of the Hamiltonian paths. In this way, Fig. 12 right illustrates a different situation where the distance from $A$ to successive points $D$ converges to the height of the triangle. Of course, this is not a Hamiltonian refinement as we have defined in this paper. In order to prove that if we use Hamiltonian refinements, we obtain always a SFC, we give the following definition.

Definition 9 (Pivot Vertex). In a $H_{k}$-triangulation of a triangle, we call pivot vertex to the vertex of the original triangle that is not an ear in the $H_{k}$-triangulation.

Theorem 10. Given a sequence of Hamiltonian refinements $\tau_{n}$, the limit of the Hamiltonian paths of $\tau_{n}$ is a SFC.

Proof. If we define the diameter of a triangle as the longest distance between two points of the triangle, i.e., the longest edge, then the result is equivalent to proving that the diameters of the triangles obtained in the refinements tend to zero [17]. For example if $k=1$, it is clear that the distance between non-pivot vertices is halved in each iteration, so we only need to check that the distances from pivot vertices decrease in such a way that we can guarantee the result. After one iteration the maximum distance from a pivot vertex could be the distance from that vertex to the middle point of the opposite edge, then we consider another iteration with the same point as a pivot vertex, by using elementary geometric arguments, the maximum distance now will be less than three quarters of the maximum distance in the original triangle. Fig. 12 left, shows an example where the distance from $A$ to $D$ is smaller than three quarters of the distance from $A$ to $B$. Then, by iterating the argument, if $d_{n}$ is the diameter after $n$ refinements, we have $d_{n} \leq|A B|\left(\frac{3}{4}\right)^{n} \longrightarrow 0$. Fig. 12 right illustrates a different situation where the distance from $A$ to successive points $D$ converges to the height of the triangle. 


\section{Conclusions and open problem}

It has been proved that for any given Hamiltonian triangulation there is a refinement which is also a Hamiltonian triangulation and besides preserves the nesting condition of the corresponding SFC's. Also the number of such Hamiltonian refinements has been bounded both from below and from above.

An interesting open problem is to tackle the tridimensional question, this is, for any Hamiltonian 3D triangulation is there a refinement which is also a Hamiltonian 3D triangulation? In 3D we can treat different Hamiltonian paths, for example the paths that enter and leave through a face, edge or vertex. An interesting result in [6] shows the existence of a through-vertex Hamiltonian path between any two tetrahedra, under certain conditions. However whether the same result is achieved or not for element-based paths and under a iterative refinement is still an open problem.

\section{References}

[1] D. Hilbert, Ueber die stetige Abbildung einer Linie auf ein Flaechenstueck, Math. Ann. 38 (1891) 459-460.

[2] M. Bader, Space-Filling Curves: An Introduction With Applications in Scientific Computing, Springer, 2013.

[3] C. Gotsman, M. Lindenbaum, On the metric properties of discrete space-filling curves, IEEE Trans. Image Process. 5 (5) (1996) $794-797$.

[4] M. Gopi, D. Eppstien, Single-strip triangulation of manifolds with arbitrary topology, Comput. Graph. Forum 23 (3) (2004) $371-379$.

[5] J.J. Bartholdi III, P. Goldsman, The vertex-adjacency dual of a triangulated irregular network has a hamiltonian cycle, Oper. Res. Lett. 32 (4) (2004) 304-308.

[6] H. Liu, L. Zhang, Existence and construction of hamiltonian paths and cycles on conforming tetrahedral meshes, Int. J. Comput. Math. 88 (6) (2011) $1137-1143$

[7] R. Pajarola, M. Antonijuan, R. Lario, Quadtin: Quadtree based triangulated irregular networks, in: Proceedings of the Conference on Visualization '02, VIS '02, IEEE Computer Society, Washington, DC, USA, 2002, pp. 395-402.

[8] W.S. Evans, D.G. Kirkpatrick, G. Townsend, Right-triangulated irregular networks, Algorithmica 30 (2) (2001) 264-286.

[9] R. Pajarola, P. Widmayer, Virtual geoexploration: Concepts and design choices, Internat. J. Comput. Geom. Appl. 11 (1)(2001) 1-14.

[10] R. Pajarola, P. Widmayer, An image compression method for spatial search, IEEE Trans. Image Process. 9 (3) (2000) 357-365.

[11] D. Burgarelli, M. Kischinhevsky, R.J. Biezuner, A new adaptive mesh refinement strategy for numerically solving evolutionary PDEO's, J. Comput. Appl. Math. 196 (1) (2006) 115-131.

[12] Á. Plaza, J.P. Suárez, M.A. Padrón, Fractality of refined triangular grids and space-filling curves, Eng. Comput. (Lond.) 20 (4) (2005) $323-332$.

[13] Á. Plaza, The eight-tetrahedra longest-edge partition and Kuhn triangulations, Comput. Math. Appl. 54 (2007) 427-433.

[14] J.P. Suárez, Á. Plaza, G.F. Carey, Propagation of longest-edge mesh patterns in local adaptive refinement, Commun. Numer. Meth. Engng. 24 (2008) $543-553$.

[15] A. Asinowski, C. Krattenthaler, T. Mansour, Counting triangulations of some classes of subdivided convex polygons, European J. Combin. 62 (2017) 92-114.

[16] F. Hurtado, M. Noy, J. Urrutia, Flipping edges in triangulations, Discrete Comput. Geom. 22 (3) (1999) 333-346.

[17] M.F. Barnsley, Fractals Everywhere, Accademic Press, 1991. 